\title{
ALGEBRAIC CONDITIONS LEADING TO CONTINUOUS LATTICES
}

\author{
JIMMIE D. LAWSON
}

\begin{abstract}
This paper is concerned with sufficient conditions for a meet-continuous lattice $L$ to be a continuous lattice. In 82 this is shown to be true if the prime elements order generate and $(L, V)$ is a compact topological semilattice. In 83 it is shown that a meet-continuous lattice with finite breadth is a continuous lattice.
\end{abstract}

In 1972 D. Scott introduced a class of lattices called continuous lattices in order to provide models for the type free calculus in logic [10]. The discovery of $\mathrm{K}$. Hofmann and A. Stralka [2] that continuous lattices are precisely the compact Hausdorff topological semilattices with identity and with a basis of subsemilattices (a class of semilattices previously studied by topological algebraists) has launched a recent flurry of activity in this area.

Proceeding from smaller classes of lattices to larger, we have the following hierarchy of lattices: (1) continuous lattices, (2) compact topological semilattices with 1 , (3) meet-continuous lattices, (4) complete lattices. In this paper we address ourselves to the general question of finding sufficient conditions on a lattice in a larger class in order that it be in a smaller class. For example Hofmann and Stralka gave necessary and sufficient algebraic conditions on a complete lattice in order that it be a continuous lattice. In [8] it was shown that a compact topological semilattice with 1 on a finite-dimensional Peano continuum or on a totally disconnected space must have a basis of subsemilattices and hence be a continuous lattice. In [7] these results were generalized to a class of spaces including those which at each point are locally homeomorphic to the product of a totally disconnected space and a finite-dimensional Peano continuum. In [6] an example was constructed showing that class (1) is a proper class of class (2). Examples that the other inclusions are strict also exist.

1. Definitions and basic results. A lattice $L$ is complete if every subset has a least upper bound and a greatest lower bound. A set $D \subset L$ is up-directed if for any $d_{1}, d_{2} \in D$, there exists $d_{3} \in D$ such that $d_{1}<d_{3}$ and $d_{2}<d_{3}$. A semilattice $S$ is meet-continuous if whenever $x=\sup D$ for an up-directed set $D$, then $x y=\sup D y$ for all $y \in D$. This definition is easily shown to be equivalent to the following conditions: if $\left\{x_{\alpha}\right\}$ in an increasing net (i.e. $\alpha<\beta$ implies $x_{\alpha}<x_{\beta}$ ) with supremum $x$ and $\left\{y_{\beta}\right\}$ is an increasing net with supremum $y$, then the increasing net $\left\{x_{\alpha} y_{\beta}\right\}$ has supremum $x y$ for all $x, y \in L$.

Received by the editors February 15, 1979 and, in revised form, May 26, 1979.

AMS (MOS) subject classifications (1970). Primary 06A20, 22A15.

Key words and phrases. Continuous lattice, topological semilattice, finite breadth. 
Let $L$ be a complete lattice and define a new relation $\ll$ on $L$ as follows: $x \ll y$ if for all up-directed sets $D$ the relation $y \leqslant \sup D$ implies the existence of a $d \in D$ with $x<d$. Equivalently $x \ll y$ if $y \leqslant \sup A$ implies that $x<\sup F$ for some finite $F \subset A$. The lattice $L$ is continuous if for all $y \in L, y=\sup \{x: x \ll y\}$. By the results of Hofmann and Stralka [2] $L$ is continuous if and only if there is a compact Hausdorff topology on $L$ such that $L$ becomes a topological semilattice relative to the multiplication $(x, y) \rightarrow x y=\inf \{x, y\}$ and has a basis of neighborhoods at each point which are subsemilattices. In this case the topology is unique and is generated by the sets $\{s \in L: x \nless s\}$ and $\{s \in L: x \ll s\}, x \in L$.

In a partially ordered set $P$, if $A \subset P$, then $\downarrow A=\{z \in P$ : $z<a$ for some $a \in A\}$ and $\uparrow A=\{y \in P: a \leqslant y$ for some $a \in A\}$. The sets $\downarrow\{x\}$ and $\uparrow\{x\}$ are denoted by $\downarrow x$ and $\uparrow y$ resp. If $S$ is a (lower) semilattice, then a subset $A \subset S$ is said to order generate $S$ if $x=\inf (A \cap \uparrow x)$ for all $x \in S$.

An element $p \in S$, a semilattice, is prime if $x y<p$ implies $x<p$ or $y<p$. Let PRIME $S$ denote the set of all primes in $S$. By [1, 3.1] a continuous lattice $S$ is distributive if and only if PRIME $S$ is order generating.

2. Distributive lattices. In this section we consider how the assumption of continuity on one of the lattice operations affects the other lattice operation. It is well known that a compact topological semilattice is a meet-continuous semilattice (see e.g. [5]). Hence this is certainly a necessary condition for topological continuity.

2.1. Proposition. Let $L$ be a distributive complete lattice equipped with a compact Hausdorff topology for which $(L, \bigvee)$ is a topological semilattice. Then with respect to this topology $(L, \wedge)$ is a topological semilattice if and only if $L$ is a meet-continuous lattice.

Proof. We have already remarked that meet-continuity is necessary. To see that it is sufficient, let $x \in L$. Then $\downarrow x$ is a compact sublattice of $L$ (since $(L, \bigvee)$ is a topological semilattice and $\downarrow x=\{y: y \vee x=x\}$ ). Since $L$ is distributive the mapping $\lambda_{x}: L \rightarrow \downarrow x$ defined by $\lambda_{x}(y)=x y$ is a lattice homomorphism which always preserves arbitrary infs. The hypothesis of meet-continuity is precisely the condition needed for $\lambda_{x}$ to preserve sups of up-directed sets, i.e., $\lambda_{x}(\sup D)=$ $\sup \left(\lambda_{x} D\right)$. Hence by [4, Theorem 15] the mapping $\lambda_{x}$ is continuous. Thus translations are continuous, i.e., multiplication in $(L, \wedge)$ is separately continuous. It follows from [3, Theorem 6] that multiplication is then jointly continuous.

2.2. RemarK. The proposition may be proved in a more direct fashion with the stronger hypothesis that $(L, \bigvee)$ is a continuous lattice. In this case $\left\{x_{\alpha}\right\}$ converges to $x$ if and only if $x$ is the lim sup of $\left\{x_{\alpha}\right\}$ and all of its subnets. The hypotheses imply that $x y$ will be the lim sup for $\left\{x_{\alpha} y_{\beta}\right\}$ and all of its subnets and hence this net will converge to $x y$. However even in this case $(L, \wedge)$ need not be a continuous lattice.

I am indebted to M. Mislove for helping me work out the details of the proof of the following proposition. 
2.3. Proposition. Let $L$ be a complete lattice equipped with a compact Hausdorff topology for which $(L, \bigvee)$ is a topological semilattice. If PRIME $L$ order generates $L$, then $L$ is a distributive continuous lattice (with respect to the meet operation).

Proof. Since PRIME $L$ order generates, $L$ is a distributive lattice. We show $L$ is meet-continuous. Let $D$ be a subset of $L$ which is directed upward, and let $x=\sup D$. For $y \in L$, we have $z=\sup (D y)<x y$. Suppose $z<x y$. Then since PRIME $L$ order generates there exists $p \in \operatorname{PRIME~} L$ such that $z \leqslant p$ and $x y \nless p$. Since $x y \leqslant y$, we have $y \leqslant p$. So $d \leqslant p$ since $d y \leqslant p$ for all $d \in D$. Hence $x=\sup D \leqslant p$, a contradiction since $x y \$ p$. Thus $z=x y$ and $L$ is meet-continuous. It follows from Proposition 2.1 that $L$ is a compact topological lattice.

To show that $L$ is a continuous lattice, we must show that $x$ has a basis of subsemilattices at each point. Let $x \in L$. By [8] it suffices to show that $x$ has a basis of subsemilattices in the sublattice $\downarrow x$. Note that the primes of $\downarrow x$ order generate $\downarrow x$, since if $p \in \operatorname{PRIME} L$, then $x p$ is prime in $\downarrow x$. This reduction essentially amounts to allowing us to assume $x=1$.

For each open set $U$ with $1 \in U$, let $z_{U}=\inf U$. Then the set $\left\{z_{U}\right\}$ is up-directed, hence converges to its supremum $y$ (since $L$ is compact). Since each $\uparrow z_{U}$ is a semilattice and a neighborhood of 1 , if $y=1$, then $\left\{\uparrow z_{U}\right\}$ would constitute a basis of subsemilattices at 1 . We assume $y \neq 1$ and derive a contradiction.

Choose open sets $U_{0}$ and $V_{0}$ such that $y \in V_{0}=\downarrow V_{0}, 1 \in U_{0}=\uparrow U_{0}$, and $\operatorname{cl}\left(U_{0}\right) \cap \operatorname{cl}\left(V_{0}\right)=\varnothing$. (We can do this since $L$ is a compact partially ordered space in the sense of Nachbin; hence $\downarrow y$ has a basis of open lower sets and 1 has a basis of open upper sets [9].) Choose open sets $A_{1}$ and $B_{1}$ such that $1 \in A_{1}, y \in B_{1}$, $A_{1} A_{1} \subset U_{0}$, and $B_{1} \vee B_{1} \subset V_{0}$. Pick open sets $U_{1}$ and $V_{1}$ such that $1 \in U_{1}=\uparrow U_{1}$, $y \in V_{1}=\downarrow V_{1}, \operatorname{cl}\left(U_{1}\right) \subset \uparrow A_{1}, \operatorname{cl}\left(V_{1}\right) \subset \downarrow B_{1}$. Note that $U_{1} U_{1} \subset U_{0}$ and $V_{1} \vee V_{1} \subset$ $V_{0}$. In this manner sequences of open sets $\left\{U_{i}: 0<i\right\}$ and $\left\{V_{i}: 0<i\right\}$ can be chosen recursively so that for each $i, 1 \in U_{i}=\uparrow U_{i}, y \in V_{i}=\downarrow V_{i}, \operatorname{cl}\left(U_{i+1}\right) \subset U_{i}$, $\operatorname{cl}\left(V_{i+1}\right) \subset V_{i}$, and $U_{i+1} U_{i+1} \subset U_{i}, V_{i+1} \vee V_{i+1} \subset V_{i}$.

Let $z_{i}=\inf U_{i}$. By the choice of $y, z_{i} \leqslant y$, and hence $z_{i} \in V_{i}$. Since $z_{i}$ is in the closure of the subsemilattice generated by $U_{i}$, we can choose $y_{i} \in V_{i}$ such that $y_{i}=\bigwedge F$ for some finite $F \subset U_{i}$. By an argument which is almost standard by now (see the proof of Theorem 8 of [4] or Proposition 6.3 of [5]) we conclude that $w=\sup \left\{y_{i}: 1 \leqslant i\right\} \in \operatorname{cl}\left(V_{0}\right)$. Also by the way the sequence $\left\{U_{i}\right\}$ was chosen, $T=\cap U_{i}=\cap \operatorname{cl}\left(U_{i}\right)$ is a compact subsemilattice, and hence has a least element $t$. Since $U_{0}=\uparrow U_{0}, \uparrow t \subset U_{0}$. Thus $w \notin \uparrow t$. Since the primes order generate, there exists a prime $p$ such that $w \leqslant p$ and $t \nless p$. Since $t \notin \downarrow p, T \cap \downarrow p=\varnothing$. Thus there exists an $i$ such that $\operatorname{cl}\left(U_{i}\right) \cap \downarrow p=\varnothing$. But there exists a finite set $F$ such that $F \subset U_{i}$ and $\inf F=y_{i} \leqslant w \leqslant p$. Since $p$ is prime $x \leqslant p$ for some $x \in F$, i.e., $U_{i} \cap \downarrow p \neq \varnothing$, a contradiction.

The distinction between complete lattices in which $(L, \wedge)$ is a compact topological semilattice and continuous lattices appears to be a rather fine one. Examples of the former that are not the latter have been few and far between (see [6]). The preceding proposition may be restated to give another set of sufficient conditions for a compact semilattice to be a continuous lattice. 
2.4. Corollary. Let $L$ be a complete lattice equipped with a compact Hausdorff topology for which $(L, \wedge)$ is a topological semilattice. If PRIME $L$ order generates and $(L, V)$ is join-continuous, then $L$ is a continuous lattice and a topological lattice.

Proof. Since PRIME $L$ order generates, $L$ is distributive. By 2.1 (with the role of the meet and join operations interchanged) $L$ is a compact topological lattice. Hence by $2.3 L$ is a continuous lattice.

RemarK. By [4] the topology on $L$ is an intrinsic topology, the so-called $C O$-topology.

In [1] it is shown that a continuous lattice $L$ which is distributive is order generated by PRIME $L$. It is unknown whether the following converse holds: Let $L$ be a compact topological semilattice with 1 in which PRIME $L$ generates. Then $L$ is continuous. Corollary 2.4 shows this conjecture is true with the additional assumption that $(L, \bigvee)$ is join-continuous.

Let $X$ be an infinite set and let $L=X \cup\{0,1\}$. Define a lattice structure on $L$ by $x y=0, x \vee y=1$, for $x, y \in X, x \neq y$. Then $L$ is a complete lattice which is a continuous lattice with respect to both of its operations. However the $C L$-topologies induced by each of the operations do not agree $(\{0\}$ is open for $(L, \bigvee)$ while $\{1\}$ is open for $(L, \wedge)$ ). By 2.1 and the uniqueness of the topology [4] the two must agree for distributive lattices. Are there other classes of interest for which they would agree?

3. Semilattices of finite breadth. A subset $A$ of a semilattice $S$ is said to be an irredundant set if for any two finite subsets $F_{1}, F_{2} \subset P, \inf F_{1}=\inf F_{2}$ implies $F_{1}=F_{2}$. Let ${ }^{N} 2$ denote the semilattice of all finite subsets of $N$, the natural numbers, under union. The singletons in ${ }^{N} 2$ form an irredundant set, and it is a straightforward exercise to show that a semilattice has a countable irredundant set if and only if it has a semilattice isomorphic to ${ }^{N_{2}}$.

The semilattice $S$ has finite breadth $n$ if $n$ is the largest cardinal such that $S$ has an irredundant subset of cardinality $n ; S$ is said to have weak finite breadth if it has no countable irredundant subset (or equivalently no isomorphic copy of ${ }^{N} 2$ ). Finite breadth implies weak finite breadth, but not conversely.

3.1. Proposition. If $S$ is a complete meet-continuous lattice, $x \nless y$, and $\sup \{z$ : $z \ll x\} \leqslant y$, then $\downarrow x \backslash \downarrow y$ contains a countable irredundant set.

Proof. First of all note that as a result of meet continuity $a \ll b$ iff $\sup D=b$ for some up-directed set implies $a \leqslant d$ for some $d \in D$.

Since not $x \ll x$, there exists a directed set $D$ with $x=\sup D$, but $x \neq d$ for all $d \in D$. Pick $x_{1} \in D$ such that $x_{1} \$ y$.

Suppose $A_{K}=\left\{x_{1}, \ldots, x_{K}\right\}$ has been chosen satisfying (i) $A_{K}$ is irredundant, and (ii) the subsemilattice $S_{K}$ generated by $A_{K}$ is a subset of $\downarrow x \backslash \downarrow y$. Let $z=$ $x_{1} x_{2} \cdots x_{K}$. Since not $z \ll x$, there exists a directed set $D$ with $x=\sup D$, but $z \nless d$ for all $d \in D$. For each $s \in S_{K}, s D$ is a directed set converging up to $s$. Since $S_{K}$ is finite, there exists $b \in D$ such that $s d \neq t d$ if $s \neq t$ for all $s, t \in S_{K}$, for all $d \geqslant b$. Since $\sup z D=z$ and $z \nless y$, there exists $p \geqslant b, p \in D$, such that 
$p z \nless y$. Let $x_{K+1}=p$. Then it is easily verified that $\left\{x_{1}, \ldots, x_{K+1}\right\}$ is irredundant and the subsemilattice this set generates is a subset of $\downarrow x \backslash \downarrow y$. Hence by recursion there exists a set with the desired properties.

3.2. Corollary. Let $S$ be a complete meet-continuous lattice satisfying the following condition: for all $x \in S, x=\sup \{y<x: \downarrow y$ has weak finite breadth $\}$. Then $S$ is a continuous lattice. In particular, a complete lattice of finite breadth is continuous if and only if it is meet-continuous.

Proof. Let $x \in S$. Let $y=\sup \{z: z \ll x\}$. If $y<x$, then there exists $q \leqslant x$ such that $\downarrow q$ has weak finite breadth and $q \nless y$. Let $p=\sup \{t: t \ll q\}$. Since $t \ll q$ implies $t \ll x$, we have $p \leqslant y$. Hence $p<q$. Now by $3.1, \downarrow q \backslash \downarrow p$ contains a countable irredundant set. However, this is impossible since $\downarrow q$ has weak finite breadth. Hence $x=y$, and thus $S$ is continuous. The second statement is immediate.

3.3. Corollary. Let $L$ be a complete lattice of weak finite breadth. Then $L$ is a distributive continuous lattice if and only if PRIME $L$ order generates $L$.

Proof. By [1] if $L$ is a distributive continuous lattice, then PRIME $L$ order generates $L$. Conversely, if PRIME $L$ order generates, then $L$ is a meet-continuous lattice (see the proof of Proposition 2.3). Hence by $3.2 L$ is a continuous lattice. The order generation of PRIME $L$ implies that $L$ is distributive.

Semilattices and lattices of finite breadth have played a major role in the study of topological semilattices and lattices (since they have close connections to the finite-dimensional ones). It is somewhat striking that such semilattices have the rather simple algebraic description of being complete and meet-continuous.

The author gratefully acknowledges support by NSF Grant MCS 76-06537 during the period of this research.

\section{REFERENCES}

1. K. H. Hofmann and J. D. Lawson, Irreducibility and generation in continuous lattices, Semigroup Forum 13 (1977), 307-353.

2. K. H. Hofmann and A. Stralka, The algebraic theory of compact Lawson semilattices-Applications of Galois connections to compact semilattices, Dissertationes Math. 137 (1976), 1-58.

3. J. D. Lawson, Additional notes on continuity in semi-topological semigroups, Semigroup Forum 12 (1976), 265-280.

4. Intrinsic topologies in topological lattices and semilattices, Pacific J. Math. 44 (1973), $593-602$.

5. __ Intrinsic lattice and semilattice topologies, Proc. 1973 Houston Lattice Theory Conf.,Univ. of Houston Press, Houston, Tex., 1973, pp. 206-260.

6. __ Lattices with no interval homomorphisms, Pacific J. Math. 32 (1970), 459-465.

7. Compact semilattices which have a basis of subsemilattices, J. London Math. Soc. (2) 16 (1977), 367-371.

8. __ Topological semilattices with small semilattices, J. London Math. Soc. 2 (1969), 719-724.

9. L. Nachbin, Topology and order, Van Nostrand, Princeton, N. J., 1965.

10. Dana Scott, Continuous lattices, toposes, algebraic geometry, and logic, Lecture Notes in Math., vol. 274, Springer-Verlag, Berlin, pp. 97-136.

Department of Mathematics, Louisiana State University, Baton Rouge, Louisiana 70803 